\title{
Existence of periodic solutions for a class of second order Hamiltonian systems
}

Da-Bin Wang ${ }^{*}$ and Kuo Yang

\begin{tabular}{l}
\hline "Correspondence: \\
wangdb96@163.com \\
Department of Applied \\
Mathematics, Lanzhou University of \\
Technology, Lanzhou, Gansu \\
730050, People's Republic of China
\end{tabular}

730050, People's Republic of China

\begin{abstract}
By using the least action principle and the minimax methods, the existence of periodic solutions for a class of second order Hamiltonian systems is considered. The results obtained in this paper extend some previous results.
\end{abstract}

Keywords: periodic solutions; second order Hamiltonian systems; least action principle

\section{Introduction and main results}

Consider the second order Hamiltonian system

$$
\left\{\begin{array}{l}
\ddot{u}(t)=\nabla F(t, u(t)), \\
u(T)-u(0)=\dot{u}(T)-\dot{u}(0)=0,
\end{array}\right.
$$

where $T>0$ and $F:[0, T] \times \mathbb{R}^{N} \rightarrow \mathbb{R}$ satisfies the following assumption:

(A) $F(t, x)$ is measurable in $t$ for every $x \in \mathbb{R}^{N}$, continuously differentiable in $x$ for a.e. $t \in[0, T]$, and there exist $a \in C\left(\mathbb{R}^{+}, \mathbb{R}^{+}\right), b \in L^{1}\left([0, T] ; \mathbb{R}^{+}\right)$such that

$$
|F(t, x)| \leq a(|x|) b(t), \quad|\nabla F(t, x)| \leq a(|x|) b(t)
$$

for all $x \in \mathbb{R}^{N}$ and a.e. $t \in[0, T]$.

The corresponding functional $\varphi: H_{T}^{1} \rightarrow \mathbb{R}$,

$$
\varphi(u)=\frac{1}{2} \int_{0}^{T}|\dot{u}(t)|^{2} d t+\int_{0}^{T} F(t, u(t)) d t,
$$

is continuously differentiable and weakly lower semi-continuous on $H_{T}^{1}$, where $H_{T}^{1}$ is the usual Sobolev space with the norm

$$
\|u\|=\left(\int_{0}^{T}|u(t)|^{2} d t+\int_{0}^{T}|\dot{u}(t)|^{2} d t\right)^{1 / 2}
$$

for $u \in H_{T}^{1}$, and

$$
\left\langle\varphi^{\prime}(u), v\right\rangle=\int_{0}^{T}[(\dot{u}(t), \dot{v})+(\nabla F(t, u(t)), v(t))] d t
$$

(c) 2015 Wang and Yang. This article is distributed under the terms of the Creative Commons Attribution 4.0 International License (http://creativecommons.org/licenses/by/4.0/), which permits unrestricted use, distribution, and reproduction in any medium, provided you give appropriate credit to the original author(s) and the source, provide a link to the Creative Commons license, and indicate if changes were made. 
for all $u, v \in H_{T}^{1}$, It is well known that the solutions of problem (1.1) correspond to the critical points of $\varphi$.

The existence of periodic solutions for problem (1.1) is obtained in [1-22] with many solvability conditions by using the least action principle and the minimax methods, such as the coercive type potential condition (see [2]), the convex type potential condition (see [5]), the periodic type potential conditions (see [16]), the even type potential condition (see [4]), the subquadratic potential condition in Rabinowitz's sense (see [9]), the bounded nonlinearity condition (see [6]), the subadditive condition (see [11]), the sublinear nonlinearity condition (see $[3,13])$, and the linear nonlinearity condition (see $[7,15,19,20])$.

In particular, when the nonlinearity $\nabla F(t, x)$ is bounded, that is, there exists $g(t) \in$ $L^{1}\left([0, T], \mathbb{R}^{+}\right)$such that $|\nabla F(t, x)| \leq g(t)$ for all $x \in \mathbb{R}^{N}$ and a.e. $t \in[0, T]$, and that

$$
\int_{0}^{T} F(t, x) d t \rightarrow \pm \infty \quad \text { as }|x| \rightarrow \infty
$$

Mawhin and Willem [6] proved that problem (1.1) has at least one periodic solution.

In $[3,13]$, Han and Tang generalized these results to the sublinear case:

$$
|\nabla F(t, x)| \leq f(t)|x|^{\alpha}+g(t) \quad \text { for all } x \in \mathbb{R}^{N} \text { and a.e. } t \in[0, T]
$$

and

$$
|x|^{-2 \alpha} \int_{0}^{T} F(t, x) d t \rightarrow \pm \infty \quad \text { as }|x| \rightarrow \infty,
$$

where $f(t), g(t) \in L^{1}\left([0, T], \mathbb{R}^{+}\right)$and $\alpha \in[0,1)$.

Subsequently, when $\alpha=1$ Zhao and Wu [19, 20] and Meng and Tang [7, 15] also proved the existence of periodic solutions for problem (1.1), i.e. $\nabla F(t, x)$ was linear:

$$
|\nabla F(t, x)| \leq f(t)|x|+g(t) \quad \text { for all } x \in \mathbb{R}^{N} \text { and a.e. } t \in[0, T],
$$

where $f(t), g(t) \in L^{1}\left([0, T], \mathbb{R}^{+}\right)$.

Recently, Wang and Zhang [21] used a control function $h(|x|)$ instead of $|x|^{\alpha}$ in (1.2) and (1.3) and got some new results, where $h$ satisfied the following conditions:

(B) $h \in C([0, \infty),[0, \infty))$ and there exist constants $C_{0}>0, K_{1}>0, K_{2}>0, \alpha \in[0,1)$ such that

(i) $h(s) \leq h(t) \forall s \leq t, s, t \in[0, \infty)$,

(ii) $h(s+t) \leq C_{0}(h(s)+h(t)) \forall s, t \in[0, \infty)$,

(iii) $0 \leq h(s) \leq K_{1} s^{\alpha}+K_{2} \forall s \in[0, \infty)$,

(iv) $h(s) \rightarrow \infty$ as $s \rightarrow \infty$.

Motivated by the results mentioned above, we will consider the periodic solutions for problem (1.1). The following are our main results.

Theorem 1.1 Suppose that $F(t, x)=F_{1}(t, x)+F_{2}(x)$, where $F_{1}$ and $F_{2}$ satisfy assumption (A) and the following conditions:

(1) there exist $f, g \in L^{1}\left([0, T] ; \mathbb{R}^{+}\right)$such that

$$
\left|\nabla F_{1}(t, x)\right| \leq f(t) h(|x|)+g(t)
$$

for all $x \in \mathbb{R}^{N}$ and a.e. $t \in[0, T]$, here $h$ satisfies (B); 
(2) there exist constants $r>0$ and $\gamma \in[0,2)$ such that

$$
\left(\nabla F_{2}(x)-\nabla F_{2}(y), x-y\right) \geq-r|x-y|^{\gamma}
$$

for all $x, y \in \mathbb{R}^{N}$

(3)

$$
\liminf _{|x| \rightarrow \infty} h^{-2}(|x|) \int_{0}^{T} F(t, x) d t>\frac{T^{2} C_{0}^{2}}{8 \pi^{2}} \int_{0}^{T} f^{2}(t) d t
$$

Then problem (1.1) has at least one periodic solution which minimizes $\varphi$ on $H_{T}^{1}$.

Theorem 1.2 Suppose that $F(t, x)=F_{1}(t, x)+F_{2}(x)$, where $F_{1}$ and $F_{2}$ satisfy assumption (A), (1), (2), and the following conditions:

(4) there exist $\delta \in[0,2)$ and $\mu>0$ such that

$$
\left(\nabla F_{2}(x)-\nabla F_{2}(y), x-y\right) \leq \mu|x-y|^{\delta}
$$

for all $x, y \in R^{N}$

(5)

$$
\limsup _{|x| \rightarrow \infty} h^{-2}(|x|) \int_{0}^{T} F(t, x) d t<-\frac{3 T^{2} C_{0}^{2}}{8 \pi^{2}} \int_{0}^{T} f^{2}(t) d t
$$

Then problem (1.1) has at least one periodic solution which minimizes $\varphi$ on $H_{T}^{1}$.

Theorem 1.3 Suppose that $F(t, x)=F_{1}(t, x)+F_{2}(x)$, where $F_{1}$ and $F_{2}$ satisfy assumption (A), (1), and the following conditions:

(6) there exists a constant $0<r<4 \pi^{2} / T^{2}$, such that

$$
\left(\nabla F_{2}(x)-\nabla F_{2}(y), x-y\right) \geq-r|x-y|^{2}
$$

for all $x, y \in R^{N}$

(7)

$$
\liminf _{|x| \rightarrow \infty} h^{-2}(|x|) \int_{0}^{T} F(t, x) d t>\frac{T^{2}}{2\left(4 \pi^{2}-r T^{2}\right)} \int_{0}^{T} f^{2}(t) d t .
$$

Then problem (1.1) has at least one periodic solution which minimizes $\varphi$ on $H_{T}^{1}$.

Theorem 1.4 Suppose that $F=F_{1}+F_{2}$, where $F_{1}$ and $F_{2}$ satisfy assumption (A), (1), and the following conditions:

(8) there exist $k \in L^{1}\left([0, T] ; \mathbb{R}^{+}\right)$and $(\lambda, \mu)$-subconvex potential $G: \mathbb{R}^{N} \rightarrow \mathbb{R}$ with $\lambda>1 / 2$ and $0<\mu<2 \lambda^{2}$, such that

$$
\left(\nabla F_{2}(t, x), y\right) \geq-k(t) G(x-y),
$$

for all $x, y \in \mathbb{R}^{N}$; 
(9)

$$
\begin{aligned}
& \limsup _{|x| \rightarrow \infty} h^{-2}(|x|) \int_{0}^{T} F_{1}(t, x) d t<-\frac{3 T^{2} C_{0}^{2}}{8 \pi^{2}} \int_{0}^{T} f^{2}(t) d t, \\
& \limsup _{|x| \rightarrow \infty}|x|^{-\beta} \int_{0}^{T} F_{2}(t, x) d t \leq-8 \mu \max _{|s| \leq 1} G(s) \int_{0}^{T} k(t) d t,
\end{aligned}
$$

where $\beta=\log _{2 \lambda}(2 \mu)$.

Then problem (1.1) has at least one periodic solution which minimizes $\varphi$ on $H_{T}^{1}$.

Remark 1.5 Theorems 1.1-1.4 extend some existing results: (i) [22], Theorems 1.1-1.4, are special cases of Theorems 1.1-1.4 with control function $h(t)=t^{\alpha}, \alpha \in[0,1), t \in[0,+\infty)$; (ii) if $F_{2}=0$, [15], Theorems 1 and 2, are special cases of Theorem 1.1 and Theorem 1.2, respectively; (iii) If $F_{2}=0$, Theorem 1.1 and Theorem 1.2 extend [21], Theorems 1.1 and 1.2 , since we weaken the so-called Ahmad-Lazer-Paul type conditions with the control function $h(t)$.

\section{Proof of theorems}

For $u \in H_{T}^{1}$, let $\bar{u}=\frac{1}{T} \int_{0}^{T}|\dot{u}(t)| d t$ and $\tilde{u}(t)=u(t)-\bar{u}$. Then one has

$$
\begin{aligned}
\|\tilde{u}\|_{\infty}^{2} & \leq \frac{T}{12} \int_{0}^{T}|\dot{u}(t)|^{2} d t \quad \text { (Sobolev's inequality) } \\
\|\tilde{u}\|_{L^{2}}^{2} & \leq \frac{T^{2}}{4 \pi^{2}} \int_{0}^{T}|\dot{u}(t)|^{2} d t \quad \text { (Wirtinger's inequality). }
\end{aligned}
$$

For the sake of convenience, we denote $M_{1}=\left(\int_{0}^{T} f^{2}(t) d t\right)^{1 / 2}, M_{2}=\int_{0}^{T} f(t) d t, M_{3}=$ $\int_{0}^{T} g(t) d t$

Proof of Theorem 1.1 Due to (3), we can choose an $a_{1}>T^{2} /\left(4 \pi^{2}\right)$ such that

$$
\liminf _{|x| \rightarrow \infty} h^{-2}(|x|) \int_{0}^{T} F(t, x) d t>\frac{a_{1} C_{0}^{2}}{2} M_{1}^{2} .
$$

For (B) and the Sobolev inequality, for any $u \in H_{T}^{1}$ we have

$$
\begin{aligned}
& \left|\int_{0}^{T}\left[F_{1}(t, u(t))-F_{1}(t, \bar{u})\right] d t\right| \\
& \quad=\left|\int_{0}^{T} \int_{0}^{1}\left(\nabla F_{1}(t, \bar{u}+s \tilde{u}(t)), \tilde{u}(t)\right) d s d t\right| \\
& \quad \leq \int_{0}^{T} \int_{0}^{1} f(t) h(|\bar{u}+s \tilde{u}(t)|)|\tilde{u}(t)| d s d t+\int_{0}^{T} \int_{0}^{1} g(t)|\tilde{u}(t)| d s d t \\
& \leq \int_{0}^{T} \int_{0}^{1} C_{0} f(t)(h(|\bar{u}|)+h(|\tilde{u}(t)|))|\tilde{u}(t)| d s d t+M_{3}\|\tilde{u}\|_{\infty} \\
& \leq C_{0} h(|\bar{u}|)\left(\int_{0}^{T} f^{2}(t) d t\right)^{1 / 2}\left(\int_{0}^{T}|\tilde{u}(t)|^{2} d t\right)^{1 / 2} \\
& \quad+C_{0} \int_{0}^{T} f(t) h(|\tilde{u}(t)|)|\tilde{u}(t)| d t+M_{3}\|\tilde{u}\|_{\infty}
\end{aligned}
$$




$$
\begin{aligned}
\leq & C_{0} M_{1} h(|\bar{u}|)\|\tilde{u}\|_{L^{2}}+C_{0} \int_{0}^{T} f(t)\left(K_{1}|\tilde{u}(t)|^{\alpha}+K_{2}\right)|\tilde{u}(t)| d t+M_{3}\|\tilde{u}\|_{\infty} \\
\leq & C_{0} M_{1} h(|\bar{u}|)\|\tilde{u}\|_{L^{2}}+C_{0} M_{2} K_{1}\|\tilde{u}\|_{\infty}^{1+\alpha}+C_{0} M_{2} K_{2}\|\tilde{u}(t)\|_{\infty}+M_{3}\|\tilde{u}(t)\|_{\infty} \\
\leq & \frac{1}{2 a_{1}}\|\tilde{u}\|_{L^{2}}^{2}+\frac{a_{1}\left(C_{0} M_{1}\right)^{2}}{2} h^{2}(|\bar{u}|)+C_{0} M_{2} K_{1}\|\tilde{u}\|_{\infty}^{1+\alpha} \\
& +C_{0} M_{2} K_{2}\|\tilde{u}(t)\|_{\infty}+M_{3}\|\tilde{u}(t)\|_{\infty} \\
\leq & \frac{T^{2}}{8 \pi^{2} a_{1}}\|\dot{u}\|_{L^{2}}^{2}+\frac{a_{1}\left(C_{0} M_{1}\right)^{2}}{2} h^{2}(|\bar{u}|)+\left(\frac{T}{12}\right)^{(1+\alpha) / 2} C_{0} M_{2} K_{1}\|\dot{u}\|_{L^{2}}^{1+\alpha} \\
& +\left(\frac{T}{12}\right)^{1 / 2} C_{0} M_{2} K_{2}\|\dot{u}\|_{L^{2}}+\left(\frac{T}{12}\right)^{1 / 2} M_{3}\|\dot{u}\|_{L^{2}} .
\end{aligned}
$$

Similarly, from (2) and the Sobolev inequality, for any $u \in H_{T}^{1}$ we get

$$
\begin{aligned}
& \int_{0}^{T}\left[F_{2}(u(t))-F_{2}(\bar{u})\right] d t \\
& =\int_{0}^{T} \int_{0}^{1} \frac{1}{s}\left(\nabla F_{2}(\bar{u}+s \tilde{u}(t))-\nabla F_{2}(\bar{u}), s \tilde{u}(t)\right) d s d t \\
& \geq-\int_{0}^{T} \int_{0}^{1} r s^{\gamma-1}|\tilde{u}(t)|^{\gamma} d s d t \\
& \geq-\frac{r T}{\gamma}\|\tilde{u}\|_{\infty}^{\gamma} \\
& \geq-\frac{r T}{\gamma}\left(\frac{T}{12}\right)^{\gamma / 2}\|\dot{u}\|_{L^{2}}^{\gamma} .
\end{aligned}
$$

From (2.2) and (2.3) we have

$$
\begin{aligned}
\varphi(u)= & \frac{1}{2}\|\dot{u}\|_{L^{2}}^{2}+\int_{0}^{T}\left[F_{1}(t, u(t))-F_{1}(t, \bar{u})\right] d t \\
& +\int_{0}^{T}\left[F_{2}(u(t))-F_{2}(\bar{u})\right] d t+\int_{0}^{T} F(t, \bar{u}) d t \\
\geq & \left(\frac{1}{2}-\frac{T^{2}}{8 \pi^{2} a_{1}}\right)\|\dot{u}\|_{L^{2}}^{2}-\frac{a_{1}\left(C_{0} M_{1}\right)^{2}}{2} h^{2}(|\bar{u}|)-\left(\frac{T}{12}\right)^{\frac{1+\alpha}{2}} C_{0} M_{2} K_{1}\|\dot{u}\|_{L^{2}}^{1+\alpha} \\
& -\left(\frac{T}{12}\right)^{1 / 2} C_{0} M_{2} K_{2}\|\dot{u}\|_{L^{2}}-\left(\frac{T}{12}\right)^{1 / 2} M_{3}\|\dot{u}\|_{L^{2}} \\
& -\frac{r T}{\gamma}\left(\frac{T}{12}\right)^{\alpha / 2}\|\dot{u}\|_{L^{2}}^{\gamma}+\int_{0}^{T} F(t, \bar{u}) d t \\
\geq & \left(\frac{1}{2}-\frac{T^{2}}{8 \pi^{2} a_{1}}\right)\|\dot{u}\|_{L^{2}}^{2}+h^{2}(|\bar{u}|)\left(h^{-2}(|\bar{u}|) \int_{0}^{T} F(t, \bar{u}) d t-\frac{a_{1}\left(C_{0} M_{1}\right)^{2}}{2}\right) \\
& -\left(\frac{T}{12}\right)^{1 / 2}\left(C_{0} M_{2} K_{2}+M_{3}\right)\|\dot{u}\|_{L^{2}} \\
& -\left(\frac{T}{12}\right)^{\frac{1+\alpha}{2}} C_{0} M_{2} K_{1}\|\dot{u}\|_{L^{2}}^{1+\alpha}-\frac{r T}{\gamma}\left(\frac{T}{12}\right)^{\gamma / 2}\|\dot{u}\|_{L^{2}}^{\gamma},
\end{aligned}
$$

for all $u \in H_{T}^{1}$. So, by (2.1) we get $\varphi(u) \rightarrow \infty$ as $\|u\| \rightarrow \infty$. 
Hence, applying the least action principle (see [6], Theorem 1.1 and Corollary 1.1), the proof is complete.

Proof of Theorem 1.2 Step 1. First, we assert that $\varphi$ satisfies the (PS) condition. Suppose that $\left\{u_{n}\right\}$ is a (PS) sequence, that is, $\varphi^{\prime}\left(u_{n}\right) \rightarrow 0$ as $n \rightarrow \infty$ and $\left\{\varphi\left(u_{n}\right)\right\}$ is bounded. For (5), we can choose an $a_{2}>T^{2} /\left(4 \pi^{2}\right)$ such that

$$
\limsup _{|x| \rightarrow \infty} h^{-2}(|x|) \int_{0}^{T} F(t, x) d t<-\left(\frac{a_{2}}{2}+\frac{\sqrt{a_{2}} T}{2 \pi}\right) C_{0}^{2} \int_{0}^{T} f^{2}(t) d t
$$

Similar to the proof of Theorem 1.1, we have

$$
\begin{aligned}
& \left|\int_{0}^{T}\left(\nabla F_{1}\left(t, u_{n}(t)\right), \tilde{u}_{n}(t)\right) d t\right| \\
& \quad \leq \frac{T^{2}}{8 \pi^{2} a_{2}}\left\|\dot{u}_{n}\right\|_{L^{2}}^{2}+\frac{a_{2}\left(C_{0} M_{1}\right)^{2}}{2} h^{2}\left(\left|\bar{u}_{n}\right|\right)+\left(\frac{T}{12}\right)^{(1+\alpha) / 2} C_{0} M_{2} K_{1}\left\|\dot{u}_{n}\right\|_{L^{2}}^{1+\alpha} \\
& \quad+\left(\frac{T}{12}\right)^{1 / 2}\left(C_{0} M_{2} K_{2}+M_{3}\right)\left\|\dot{u}_{n}\right\|_{L^{2}}
\end{aligned}
$$

and

$$
\int_{0}^{T}\left(\nabla F_{2}\left(u_{n}(t)\right), \tilde{u}_{n}(t)\right) d t \geq-\frac{r T}{\gamma}\left(\frac{T}{12}\right)^{\gamma / 2}\|\dot{u}\|_{L^{2}}^{\gamma}
$$

for all $n$. Hence we have

$$
\begin{aligned}
\left\|\tilde{u}_{n}\right\| \geq & \left\langle\varphi^{\prime}\left(u_{n}\right), \tilde{u}_{n}\right\rangle \\
= & \left\|\dot{u}_{n}\right\|_{L^{2}}^{2}+\int_{0}^{T}\left(\nabla F\left(t, u_{n}(t)\right), \tilde{u}_{n}(t)\right) d t \\
\geq & \left(1-\frac{T^{2}}{8 \pi^{2} a_{2}}\right)\left\|\dot{u}_{n}\right\|_{L^{2}}^{2}-\frac{a_{2}\left(C_{0} M_{1}\right)^{2}}{2} h^{2}\left(\left|\bar{u}_{n}\right|\right) \\
& -\left(\frac{T}{12}\right)^{\frac{1+\alpha}{2}} C_{0} M_{2} K_{1}\left\|\dot{u}_{n}\right\|_{L^{2}}^{1+\alpha} \\
& -\left(\frac{T}{12}\right)^{1 / 2}\left(C_{0} M_{2} K_{2}+M_{3}\right)\left\|\dot{u}_{n}\right\|_{L^{2}}-\frac{r T}{\gamma}\left(\frac{T}{12}\right)^{\gamma / 2}\left\|\dot{u}_{n}\right\|_{L^{2}}^{\gamma},
\end{aligned}
$$

for large $n$. So, by Wirtinger's inequality we get

$$
\left\|\left(\tilde{u}_{n}\right)\right\| \leq \frac{\left(T^{2}+4 \pi^{2}\right)^{1 / 2}}{2 \pi}\left\|\dot{u}_{n}\right\|_{L^{2}}
$$

From (2.6) and (2.7),

$$
\begin{aligned}
& \frac{a_{2}\left(C_{0} M_{1}\right)^{2}}{2} h^{2}\left(\left|\bar{u}_{n}\right|\right) \\
& \quad \geq\left(1-\frac{T^{2}}{8 \pi^{2} a_{2}}\right)\left\|\dot{u}_{n}\right\|_{L^{2}}^{2}-C_{0} M_{2} K_{1}\left(\frac{T}{12}\right)^{\frac{1+\alpha}{2}}\left\|\dot{u}_{n}\right\|_{L^{2}}^{1+\alpha}
\end{aligned}
$$




$$
\begin{aligned}
& -\left(\frac{T}{12}\right)^{1 / 2}\left(C_{0} M_{2} K_{2}+M_{3}\right)\left\|\dot{u}_{n}\right\|_{L^{2}} \\
& -\left\|\tilde{u}_{n}\right\|-\frac{r T}{\gamma}\left(\frac{T}{12}\right)^{\gamma / 2}\left\|\dot{u}_{n}\right\|_{L^{2}}^{\gamma} \geq \frac{1}{2}\left\|\dot{u}_{n}\right\|_{L^{2}}^{2}+C_{1},
\end{aligned}
$$

where

$$
\begin{aligned}
C_{1}= & \min _{s \in[0,+\infty]}\left\{\frac{4 \pi^{2} a_{2}-T^{2}}{8 \pi^{2} a_{2}} s^{2}-\left(\frac{T}{12}\right)^{\frac{1+\alpha}{2}} C_{0} M_{2} K_{1} s^{1+\alpha}\right. \\
& \left.-\left[\frac{\left(T^{2}+4 \pi^{2}\right)^{1 / 2}}{2 \pi}+C_{0} M_{2} K_{2}\left(\frac{T}{12}\right)^{1 / 2}+\left(\frac{T}{12}\right)^{1 / 2} M_{3}\right] s-\frac{r T}{\gamma}\left(\frac{T}{12}\right)^{\gamma / 2} s^{\gamma}\right\} .
\end{aligned}
$$

Note that $a_{2}>T^{2} /\left(4 \pi^{2}\right)$ implies $-\infty<C_{1}<0$. Hence, it follows from (2.8) that

$$
\left\|\dot{u}_{n}\right\|_{L^{2}}^{2} \leq a_{2} C_{0}^{2} M_{1}^{2} h^{2}\left(\left|\bar{u}_{n}\right|\right)-2 C_{1}
$$

and then

$$
\left\|\dot{u}_{n}\right\|_{L^{2}} \leq \sqrt{a_{2}} C_{0} M_{1} h\left(\left|\bar{u}_{n}\right|\right)+C_{2}
$$

where $0<C_{2}<+\infty$. Similar to the proof of Theorem 1.1, we have

$$
\begin{aligned}
& \left|\int_{0}^{T}\left[F_{1}\left(t, u_{n}(t)\right)-F_{1}\left(t, \bar{u}_{n}\right)\right] d t\right| \\
& \quad \leq C_{0} M_{1} h\left(\left|\bar{u}_{n}\right|\right)\left\|\tilde{u}_{n}\right\|_{L^{2}}+C_{0} M_{2} K_{1}\left\|\tilde{u}_{n}\right\|_{\infty}^{1+\alpha}+\left(C_{0} M_{2} K_{2}+M_{3}\right)\left\|\tilde{u}_{n}\right\|_{\infty} \\
& \quad \leq \frac{\pi}{\sqrt{a_{2}} T}\left\|\tilde{u}_{n}\right\|_{L^{2}}^{2}+\frac{\sqrt{a_{2}} T C_{0}^{2}}{4 \pi} M_{1}^{2} h^{2}\left(\left|\bar{u}_{n}\right|\right) \\
& \quad+C_{0} M_{2} K_{1}\left\|\tilde{u}_{n}\right\|_{\infty}^{1+\alpha}+\left(C_{0} M_{2} K_{2}+M_{3}\right)\left\|\tilde{u}_{n}\right\|_{\infty} \\
& \leq \frac{T}{4 \pi \sqrt{a_{2}}}\left\|\dot{u}_{n}\right\|_{L^{2}}^{2}+\frac{\sqrt{a_{2}} T C_{0}^{2}}{4 \pi} M_{1}^{2} h^{2}\left(\left|\bar{u}_{n}\right|\right)+\left(\frac{T}{12}\right)^{(1+\alpha) / 2} C_{0} M_{2} K_{1}\left\|\dot{u}_{n}\right\|_{L^{2}}^{1+\alpha} \\
& \quad+\left(\frac{T}{12}\right)^{1 / 2}\left(C_{0} M_{2} K_{2}+M_{3}\right)\left\|\dot{u}_{n}\right\|_{L^{2} .}
\end{aligned}
$$

By (4), we obtain

$$
\begin{aligned}
& \int_{0}^{T}\left[F_{2}\left(u_{n}(t)\right)-F_{2}\left(\bar{u}_{n}\right)\right] d t \\
& \quad=\int_{0}^{T} \int_{0}^{1} \frac{1}{s}\left(\nabla F_{2}\left(\bar{u}_{n}+s \tilde{u}_{n}(t)\right)-\nabla F_{2}\left(\bar{u}_{n}\right), s \tilde{u}_{n}(t)\right) d s d t \\
& \quad \leq \int_{0}^{T} \int_{0}^{1} \mu s^{\delta-1}\left|\tilde{u}_{n}(t)\right|^{\delta} d s d t \leq \frac{\mu T}{\delta}\left\|\tilde{u}_{n}\right\|_{\infty}^{\delta} \\
& \quad \leq \frac{\mu T}{\delta}\left(\frac{T}{12}\right)^{\delta / 2}\left\|\dot{u}_{n}\right\|_{L^{2}}^{\delta} .
\end{aligned}
$$


From the boundedness of $\varphi\left(u_{n}\right)$ and (2.9)-(2.11), we have

$$
\begin{aligned}
& C_{3} \leq \varphi\left(u_{n}\right) \\
& =\frac{1}{2}\left\|\dot{u}_{n}\right\|_{L^{2}}^{2}+\int_{0}^{T}\left[F_{1}\left(t, u_{n}(t)\right)-F_{1}\left(t, \bar{u}_{n}\right)\right] d t+\int_{0}^{T}\left[F_{2}\left(u_{n}(t)\right)-F_{2}\left(\bar{u}_{n}\right)\right] d t \\
& +\int_{0}^{T} F\left(t, \bar{u}_{n}(t)\right) d t \\
& \leq\left(\frac{1}{2}+\frac{T}{4 \pi \sqrt{a_{2}}}\right)\|\dot{u}\|_{L^{2}}^{2}+\frac{\sqrt{a_{2}} T C_{0}^{2}}{4 \pi} M_{1}^{2} h^{2}(|\bar{u}|)+C_{0} M_{2} K_{1}\left(\frac{T}{12}\right)^{(1+\alpha) / 2}\left\|\dot{u}_{n}\right\|_{L^{2}}^{1+\alpha} \\
& +\left(\frac{T}{12}\right)^{1 / 2}\left(C_{0} M_{2} K_{2}+M_{3}\right)\left\|\dot{u}_{n}\right\|_{L^{2}}+\int_{0}^{T} F\left(t, \bar{u}_{n}\right) d t+\frac{\mu T}{\delta}\left(\frac{T}{12}\right)^{\delta / 2}\left\|\dot{u}_{n}\right\|_{L^{2}}^{\delta} \\
& \leq\left(\frac{1}{2}+\frac{T}{4 \pi \sqrt{a_{2}}}\right)\left(a_{2} C_{0}^{2} M_{1}^{2} h^{2}\left(\left|\bar{u}_{n}\right|\right)-2 C_{1}\right)+\frac{\sqrt{a_{2}} T C_{0}^{2}}{4 \pi} M_{1}^{2} h^{2}(|\bar{u}|) \\
& +C_{0} M_{2} K_{1}\left(\frac{T}{12}\right)^{(1+\alpha) / 2}\left(\sqrt{a_{2}} C_{0} M_{1} h\left(\left|\bar{u}_{n}\right|\right)+C_{2}\right)^{1+\alpha} \\
& +\left(\frac{T}{12}\right)^{1 / 2}\left(C_{0} M_{2} K_{2}+M_{3}\right)\left(\sqrt{a_{2}} C_{0} M_{1} h\left(\left|\bar{u}_{n}\right|\right)+C_{2}\right) \\
& +\int_{0}^{T} F\left(t, \bar{u}_{n}\right) d t+\frac{\mu T}{\delta}\left(\frac{T}{12}\right)^{\delta / 2}\left\|\dot{u}_{n}\right\|_{L^{2}}^{\delta} \\
& \leq\left(\frac{a_{2}}{2}+\frac{\sqrt{a_{2}} T}{2 \pi}\right) C_{0}^{2} M_{1}^{2} h^{2}\left(\left|\bar{u}_{n}\right|\right)-\left(1+\frac{T}{2 \pi \sqrt{a_{2}}}\right) C_{1} \\
& +\left(\frac{T}{12}\right)^{(1+\alpha) / 2} 2^{\alpha} C_{0} M_{2} K_{1}\left[\left(\sqrt{a_{2}} C_{0} M_{1}\right)^{1+\alpha} h\left(\left|\bar{u}_{n}\right|\right)^{1+\alpha}+C_{2}^{1+\alpha}\right] \\
& +\left(\frac{T}{12}\right)^{1 / 2}\left(C_{0} M_{2} K_{2}+M_{3}\right)\left(\sqrt{a_{2}} C_{0} M_{1} h\left(\left|\bar{u}_{n}\right|\right)+C_{2}\right) \\
& +\int_{0}^{T} F\left(t, \bar{u}_{n}\right) d t+\frac{\mu T}{\delta}\left(\frac{T}{12}\right)^{\delta / 2} 2^{\delta-1}\left(\left(\sqrt{a_{2}} M_{1}\right)^{\delta} h^{\delta}\left(\left|\bar{u}_{n}\right|\right)+C_{2}^{\delta}\right) \\
& =h^{2}\left(\left|\bar{u}_{n}\right|\right)\left[h^{-2}\left(\left|\bar{u}_{n}\right|\right) \int_{0}^{T} F\left(t, \bar{u}_{n}\right) d t+\left(\frac{a_{2}}{2}+\frac{\sqrt{a_{2}} T}{2 \pi}\right) C_{0}^{2} M_{1}^{2}\right. \\
& +\left(\frac{T}{12}\right)^{(1+\alpha) / 2} 2^{\alpha} C_{0}^{2+\alpha} M_{2} M_{1}^{1+\alpha} K_{1} h^{\alpha-1}\left(\left|\bar{u}_{n}\right|\right)+\left(\frac{T}{12}\right)^{1 / 2}\left(C_{0} M_{2} K_{2}\right. \\
& \left.\left.+M_{3}\right)\left(\sqrt{a_{2}} C_{0} M_{1} h^{-1}\left(\left|\bar{u}_{n}\right|\right)\right)+\frac{\mu T}{\delta}\left(\frac{T}{12}\right)^{\delta / 2} 2^{\delta-1}\left(\sqrt{a_{2}} M_{1}\right)^{\delta} h^{\delta-2}\left(\left|\bar{u}_{n}\right|\right)\right] \\
& +\left(\frac{T}{12}\right)^{(1+\alpha) / 2} 2^{\alpha} C_{0} M_{2} K_{1} C_{2}^{1+\alpha}+\left(\frac{T}{12}\right)^{1 / 2}\left(C_{0} M_{2} K_{2}\right. \\
& \left.+M_{3}\right) C_{2}-\left(1+\frac{T}{2 \pi \sqrt{a_{2}}}\right) C_{1}+\frac{\mu T}{\delta}\left(\frac{T}{12}\right)^{\delta / 2} 2^{\delta-1} C_{2}^{\delta},
\end{aligned}
$$

for large $n$. So, by (2.4) we see that $|\bar{u}|$ is bounded. Hence $\left\{u_{n}\right\}$ is bounded by (2.9). Arguing as in the proof of Proposition 4.1 of [6], we conclude that the (PS) condition is satisfied. 
Step 2. Let $\tilde{H}_{T}^{1}=\left\{u \in H_{T}^{1}: \bar{u}=0\right\}$. We assert that for $u \in \tilde{H}_{T}^{1}$,

$$
\varphi(u) \rightarrow+\infty, \quad\|u\| \rightarrow \infty
$$

In fact, from (1) and Sobolev's inequality, we get

$$
\begin{aligned}
& \left|\int_{0}^{T}\left[F_{1}(t, u(t))-F_{1}(t, 0)\right] d t\right| \\
& \quad=\left|\int_{0}^{T} \int_{0}^{1}(\nabla F(t, s u(t)), u(t)) d s d t\right| \\
& \quad \leq \int_{0}^{T} f(t) h(|u(t)|)|u(t)| d t+\int_{0}^{T} g(t)|u(t)| d t \\
& \quad \leq \int_{0}^{T} f(t)\left(K_{1}|u(t)|^{\alpha}+K_{2}\right)|u(t)| d t+M_{3}\|u\|_{\infty} \\
& \quad \leq M_{2} K_{1}\|u\|_{\infty}^{1+\alpha}+M_{2} K_{2}\|u\|_{\infty}+M_{3}\|u\|_{\infty} \\
& \quad \leq\left(\frac{T}{12}\right)^{\frac{1+\alpha}{2}} M_{2} K_{1}\|\dot{u}\|_{L^{2}}^{1+\alpha}+\left(\frac{T}{12}\right)^{1 / 2}\left(M_{2} K_{2}+M_{3}\right)\|\dot{u}\|_{L^{2}},
\end{aligned}
$$

for all $u \in \tilde{H}_{T}^{1}$. It follows from (2) that

$$
\begin{aligned}
& \int_{0}^{T}\left[F_{2}(u(t))-F_{2}(0)\right] d t \\
& \quad=\int_{0}^{T} \int_{0}^{1} \frac{1}{s}\left(\nabla F_{2}(s \tilde{u}(t))-\nabla F_{2}(0), s u(t)\right) d s d t \\
& \quad \geq-\int_{0}^{T} \int_{0}^{1} r s^{\gamma-1}|u|^{\gamma} d s d t \\
& \quad \geq-\frac{r T}{\gamma}\|\dot{u}\|_{\infty}^{\gamma} \\
& \quad \geq-\frac{r T}{\gamma}\left(\frac{T}{12}\right)^{\gamma / 2}\|\dot{u}\|_{L^{2}}^{\gamma} .
\end{aligned}
$$

So, we get

$$
\begin{aligned}
\varphi(u)= & \frac{1}{2}\|\dot{u}\|_{L^{2}}^{2}+\int_{0}^{T}[F(t, u(t))-F(t, 0)] d t+\int_{0}^{T} F(t, 0) d t \\
\geq & \frac{1}{2}\|\dot{u}\|_{L^{2}}^{2}-\left(\frac{T}{12}\right)^{\frac{1+\alpha}{2}} M_{2} K_{1}\|\dot{u}\|_{L^{2}}^{1+\alpha}-\left(\frac{T}{12}\right)^{1 / 2}\left(M_{2} K_{2}+M_{3}\right)\|\dot{u}\|_{L^{2}} \\
& -\frac{r T}{\gamma}\left(\frac{T}{12}\right)^{\gamma / 2}\|\dot{u}\|_{L^{2}}^{\gamma}+\int_{0}^{T} F(t, 0) d t .
\end{aligned}
$$

By Wirtinger's inequality, $\|u\| \rightarrow \infty$ if and only if $\|\dot{u}\|_{L^{2}} \rightarrow \infty$ in $\tilde{H}_{T}^{1}$. Hence (2.12) holds.

Step 3. By (5), we can easily see that $\int_{0}^{T} F(t, x) d t \rightarrow-\infty$ as $|x| \rightarrow \infty$ for all $x \in \mathbb{R}^{N}$. Thus, for all $u \in\left(\tilde{H}_{T}^{1}\right)^{\perp}=\mathbb{R}^{N}$,

$$
\varphi(u)=\int_{0}^{T} F(t, u) d t \rightarrow-\infty \quad \text { as }|u| \rightarrow \infty .
$$


Now, by saddle point theorem (see, [10], Theorem 4.6), the proof is completed.

Proof of Theorem 1.3 By (7), we can choose an $a_{3}>\frac{T^{2}}{4 \pi^{2}-r T^{2}}$ such that

$$
\liminf _{|x| \rightarrow \infty} h^{-2}(|x|) \int_{0}^{T} F(t, x) d t>\frac{a_{3}}{2} M_{1}^{2} C_{0}^{2}
$$

By (6) and the Sobolev inequality, we have

$$
\begin{aligned}
& \int_{0}^{T}\left[F_{2}(u(t))-F_{2}(\bar{u})\right] d t \\
& \quad=\int_{0}^{T} \int_{0}^{1} \frac{1}{s}\left(\nabla F_{2}(\bar{u}+s \tilde{u}(t))-\nabla F_{2}(\bar{u}), s \tilde{u}(t)\right) d s d t \\
& \quad \geq-\int_{0}^{T} \int_{0}^{1} r s|\tilde{u}(t)|^{2} d s d t \geq-\frac{r T^{2}}{8 \pi^{2}}\|\dot{u}\|_{L^{2}}^{2} .
\end{aligned}
$$

By a similar method to that of the proof of Theorem 1.1, we get

$$
\begin{aligned}
\varphi(u)= & \frac{1}{2}\|\dot{u}\|_{L^{2}}^{2}+\int_{0}^{T} F(t, u(t)) d t \\
= & \frac{1}{2}\|\dot{u}\|_{L^{2}}^{2}+\int_{0}^{T}\left[F_{1}(t, u(t))-F_{1}(t, \bar{u})\right] d t \\
& +\int_{0}^{T}\left[F_{2}(u(t))-F_{2}(\bar{u})\right] d t+\int_{0}^{T} F(t, \bar{u}) d t \\
\geq & \left(\frac{1}{2}-\frac{T^{2}}{8 \pi^{2} a_{3}}-\frac{r T^{2}}{8 \pi^{2}}\right)\|\dot{u}\|_{L^{2}}^{2}-\left(\frac{T}{12}\right)^{(1+\alpha) / 2} C_{0} M_{2} K_{1}\|\dot{u}\|_{L_{2}}^{1+\alpha} \\
& -\left(\frac{T}{12}\right)^{1 / 2}\left(M_{3}+\frac{C_{0} M_{2} K_{2}}{2}\right)\|\dot{u}\|_{L^{2}}-\frac{a_{3} C_{0}^{2} M_{1}^{2}}{2} h^{2}(|\bar{u}|)+\int_{0}^{T} F(t, \bar{u}) d t \\
= & \left(\frac{1}{2}-\frac{T^{2}}{8 \pi^{2} a_{3}}-\frac{r T^{2}}{8 \pi^{2}}\right)\|\dot{u}\|_{L^{2}}^{2}-\left(\frac{T}{12}\right)^{1 / 2}\left(M_{3}+\frac{C_{0} M_{2} K_{2}}{2}\right)\|\dot{u}\|_{L^{2}} \\
& -\left(\frac{T}{12}\right)^{(1+\alpha) / 2} C_{0} M_{2} K_{1}\|\dot{u}\|_{L_{2}}^{1+\alpha}+h^{2}(|\bar{u}|)\left(h^{-2}(|\bar{u}|) \int_{0}^{T} F(t, \bar{u}) d t-\frac{a_{3} C_{0}^{2} M_{1}^{2}}{2}\right),
\end{aligned}
$$

for all $u \in H_{T}^{1}$, which implies that $\varphi(u) \rightarrow \infty$ as $\|u\| \rightarrow \infty$ by (2.13), due to the facts that $r<\frac{4 \pi^{2}}{T^{2}}$ and $\|u\| \rightarrow \infty$ if and only if $\left(|\bar{u}|^{2}+\|\dot{u}\|_{L^{2}}^{2}\right)^{1 / 2} \rightarrow \infty$. So, applying the least action principle, Theorem 1.3 holds.

Proof of Theorem 1.4 First, we assert that $\varphi$ satisfies the (PS) condition. Suppose that $\left\{u_{n}\right\}$ satisfies $\varphi^{\prime}\left(u_{n}\right) \rightarrow 0$ as $n \rightarrow \infty$ and $\left\{\varphi\left(u_{n}\right)\right\}$ is bounded. By (9), we can choose an $a_{4}>\frac{T^{2}}{4 \pi^{2}}$ such that

$$
\limsup _{|x| \rightarrow \infty} h^{-2}(|x|) \int_{0}^{T} F_{1}(t, x) d t<-\left(\frac{a_{4}}{2}+\frac{\sqrt{a_{4}} T}{2 \pi}\right) C_{0}^{2} M_{1}^{2} .
$$

By the $(\lambda, \mu)$-subconvexity of $G(x)$, we have

$$
G(x) \leq\left(2 \mu|x|^{\beta}+1\right) G_{0}
$$


for all $x \in \mathbb{R}^{N}$, and a.e. $t \in[0, T]$, where $G_{0}=\max _{|s| \leq 1} G(s), \beta=\log _{2 \lambda}(2 \mu)<2$ Then

$$
\begin{aligned}
& \int_{0}^{T}\left(\nabla F_{2}\left(t, u_{n}(t)\right), \tilde{u}_{n}(t)\right) d t \\
& \quad \geq-\int_{0}^{T} k(t) G\left(\bar{u}_{n}\right) d t \\
& \quad \geq-\int_{0}^{T} k(t)\left(2 \mu\left|\bar{u}_{n}\right|^{\beta}+1\right) G_{0} d t \\
& \quad=-2 \mu M_{4}\left|\bar{u}_{n}\right|^{\beta}-M_{4},
\end{aligned}
$$

where $M_{4}=G_{0} \int_{0}^{T} k(t) d t$. From (2.5) and (2.16), for large $n$, we have

$$
\begin{aligned}
\left\|\bar{u}_{n}\right\| \geq & \left\langle\varphi\left(u_{n}\right), \tilde{u}_{n}\right\rangle \\
= & \left\|\dot{u}_{n}\right\|_{L^{2}}^{2}+\int_{0}^{T}\left(\nabla F\left(t, u_{n}(t)\right), \tilde{u}_{n}(t)\right) \\
\geq & \left(1-\frac{T^{2}}{8 \pi^{2} a_{4}}\right)\left\|\dot{u}_{n}\right\|_{L^{2}}^{2}-\left(\frac{T}{12}\right)^{(1+\alpha) / 2} C_{0} M_{2} K_{1}\left\|\dot{u}_{n}\right\|_{L^{2}}^{1+\alpha}-\frac{\left(C_{0} M_{1}\right)^{2} a_{4}}{2} h^{2}\left(\left|\bar{u}_{n}\right|\right) \\
& -\left(\frac{T}{12}\right)^{1 / 2}\left(M_{3}+\frac{C_{0} M_{2} K_{2}}{2}\right)\|\dot{u}\|_{L^{2}}-2 \mu M_{4}\left|\bar{u}_{n}\right|^{\beta}-M_{4} .
\end{aligned}
$$

So, from (2.7) and (2.17) we have

$$
\begin{aligned}
\frac{\left(C_{0} M_{1}\right)^{2} a_{4}}{2} h^{2}\left(\left|\bar{u}_{n}\right|\right)+2 \mu M_{4}\left|\bar{u}_{n}\right|^{\beta} & \\
\geq & \left(1-\frac{T^{2}}{8 \pi^{2} a_{4}}\right)\left\|\dot{u}_{n}\right\|_{L^{2}}^{2}-\left(\frac{T}{12}\right)^{(1+\alpha) / 2} C_{0} M_{2} K_{1}\left\|\dot{u}_{n}\right\|_{L^{2}}^{1+\alpha} \\
& \quad-\left(\frac{T}{12}\right)^{1 / 2}\left(M_{3}+\frac{C_{0} M_{2} K_{2}}{2}\right)\|\dot{u}\|_{L^{2}}-\frac{\left(T^{2}+4 \pi^{2}\right)^{1 / 2}}{2 \pi}\left\|\dot{u}_{n}\right\|_{L^{2}}-M_{4} \\
\geq & \frac{1}{2}\left\|\dot{u}_{n}\right\|_{L^{2}}^{2}+C_{4},
\end{aligned}
$$

where

$$
\begin{aligned}
C_{4}= & \min \left\{\left(\frac{1}{2}-\frac{T^{2}}{8 \pi^{2} a_{4}}\right) s^{2}-\left(\frac{T}{12}\right)^{(1+\alpha) / 2} C_{0} M_{2} K_{1} s^{1+\alpha}-\left[\frac{\left(T^{2}+4 \pi^{2}\right)^{1 / 2}}{2 \pi}\right.\right. \\
& \left.\left.+\left(\frac{T}{12}\right)^{1 / 2}\left(M_{3}+\frac{C_{0} M_{2} K_{2}}{2}\right)\right] s-M_{4}\right\} .
\end{aligned}
$$

Note that $-\infty<C_{4}<0$ due to $a_{4}>\frac{T^{2}}{4 \pi^{2}}$, by (2.18), one has

$$
\left\|\dot{u}_{n}\right\|_{L^{2}}^{2} \leq a_{4}\left(C_{0} M_{1}\right)^{2} h^{2}\left(\left|\bar{u}_{n}\right|\right)+4 \mu M_{4}\left|\bar{u}_{n}\right|^{\beta}-2 C_{4},
$$

and then

$$
\left\|\dot{u}_{n}\right\|_{L^{2}} \leq \sqrt{a_{4}} C_{0} M_{1} h\left(\left|\bar{u}_{n}\right|\right)+2 \sqrt{\mu M_{4}}\left|\bar{u}_{n}\right|^{\beta / 2}+C_{5},
$$


where $C_{5}>0$. From (8) and (2.15), we have

$$
\begin{aligned}
& \left|\int_{0}^{T}\left[F_{2}(t, u(t))-F_{2}(t, \bar{u})\right] d t\right| \\
& \quad=\int_{0}^{T} \int_{0}^{1}\left(\nabla F_{2}\left(t, \bar{u}_{n}+s \tilde{u}_{n}(t)\right), \tilde{u}_{n}(t)\right) d s d t \\
& \quad \leq \int_{0}^{T} \int_{0}^{1} k(t) G\left(\bar{u}_{n}+(s+1) \tilde{u}_{n}\right) d s d t \\
& \quad \leq \int_{0}^{T} \int_{0}^{1} k(t)\left(2 \mu\left|\bar{u}_{n}+(s+1) \tilde{u}_{n}(t)\right|^{\beta}+1\right) \\
& \quad \leq 4 \mu \int_{0}^{T} k(t)\left(\left|\bar{u}_{n}\right|^{\beta}+2^{\beta}\left|\tilde{u}_{n}\right|^{\beta}\right) G_{0} \int_{0}^{T} k(t) d t \\
& \quad \leq\left(\frac{T}{12}\right)^{\beta / 2} 2^{\beta+2} \mu M_{4}\left\|\dot{u}_{n}\right\|_{L^{2}}^{\beta}+4 \mu M_{4}\left|\bar{u}_{n}\right|^{\beta}+M_{4},
\end{aligned}
$$

for all $u \in H_{T}^{1}$. By the boundedness of $\left\{\varphi\left(u_{n}\right)\right\}$ and the inequalities (2.19)-(2.21), we get

$$
\begin{aligned}
C_{6} \leq & \varphi\left(u_{n}\right) \\
= & \frac{1}{2}\left\|\dot{u}_{n}\right\|_{L^{2}}^{2}+\int_{0}^{T}\left[F_{1}\left(t, u_{n}(t)\right)-F_{1}\left(t, \bar{u}_{n}\right)\right] d t \\
& +\int_{0}^{T}\left[F_{2}\left(t, u_{n}(t)\right)-F_{2}\left(t, \bar{u}_{n}\right)\right] d t+\int_{0}^{T} F\left(t, \bar{u}_{n}\right) d t \\
\leq & \left(\frac{1}{2}+\frac{T}{4 \pi \sqrt{a_{4}}}\right)\left\|\dot{u}_{n}\right\|_{L^{2}}^{2}+\frac{\sqrt{a_{4}} T C_{0}^{2}}{4 \pi} M_{1}^{2} h^{2}\left(\left|\bar{u}_{n}\right|\right)+\left(\frac{T}{12}\right)^{1 / 2}\left(C_{0} M_{2} K_{2}+M_{3}\right)\left\|\dot{u}_{n}\right\|_{L^{2}} \\
& +\left(\frac{T}{12}\right)^{(1+\alpha) / 2} C_{0} M_{2} K_{1}\left\|\dot{u}_{n}\right\|_{L^{2}}^{1+\alpha}+\left(\frac{T}{12}\right)^{\beta / 2} 2^{\beta+2} \mu M_{4}\|\dot{u}\|_{L^{2}}^{\beta} \\
& +4 \mu M_{4}\left|\bar{u}_{n}\right|^{\beta}+M_{4}+\int_{0}^{T} F\left(t, \bar{u}_{n}\right) d t \\
\leq & \left(\frac{1}{2}+\frac{T}{4 \pi \sqrt{a_{4}}}\right)\left(a_{4}\left(C_{0} M_{1}\right)^{2} h^{2}\left(\left|\bar{u}_{n}\right|\right)+4 \mu M_{4}\left|\bar{u}_{n}\right|^{\beta}-2 C_{4}\right) \\
& +\frac{\sqrt{a_{4}} T C_{0}^{2}}{4 \pi} M_{1}^{2} h^{2}\left(\left|\bar{u}_{n}\right|\right) \\
& +\left(\frac{T}{12}\right)^{(1+\alpha) / 2} C_{0} M_{2} K_{1}\left(\sqrt{a_{4}} C_{0} M_{1} h\left(\left|\bar{u}_{n}\right|\right)+2 \sqrt{\mu M_{4}}\left|\bar{u}_{n}\right|^{\beta / 2}+C_{5}\right)^{1+\alpha} \\
\leq & \left(\frac{a_{4}}{2}+\frac{\sqrt{a_{4}} T}{2 \pi}\right)\left(\left(C_{0} M_{1}\right)^{2} h^{2}\left(\left|\bar{u}_{n}\right|\right)\right) \\
& +\left(\frac{T}{12}\right)^{1 / 2}\left(C_{0} M_{2} K_{2}+M_{3}\right)\left(\sqrt{a_{4}} C_{0} M_{1} h\left(\left|\bar{u}_{n}\right|\right)+2 \sqrt{\mu M_{4}}\left|\bar{u}_{n}\right|^{\beta / 2}+C_{5}\right) \\
& +\left(\frac{T}{12}\right)^{\beta / 2} 2^{\beta+2} \mu M_{4}\left(\sqrt{a_{4}} C_{0} M_{1} h\left(\left|\bar{u}_{n}\right|\right)+2 \sqrt{\mu M_{4}}\left|\bar{u}_{n}\right|^{\beta / 2}+C_{5}\right)^{\beta} \\
& 4 \mu M_{4}\left|\bar{u}_{n}\right|^{\beta}+M_{4}+\int_{0}^{T} F\left(t, \bar{u}_{n}\right) d t \\
&
\end{aligned}
$$




$$
\begin{aligned}
& +\left(6+\frac{T}{\pi \sqrt{a_{4}}}\right) \mu M_{4}\left|\bar{u}_{n}\right|^{\beta}-\left(1+\frac{T}{2 \pi \sqrt{a_{4}}}\right) C_{4} \\
& +\left(\frac{T}{12}\right)^{(1+\alpha) / 2} C_{0} M_{2} K_{1}\left(2^{\alpha} a_{4}^{(1+\alpha) / 2}\left(C_{0} M_{1}\right)^{1+\alpha} h^{1+\alpha}\left(\left|\bar{u}_{n}\right|\right)\right. \\
& \left.+2^{3 \alpha+1} \mu^{\frac{1+\alpha}{2}} M_{4}^{\frac{1+\alpha}{2}}\left|\bar{u}_{n}\right|^{\beta(1+\alpha)}+2^{2 \alpha} C_{5}^{1+\alpha}\right) \\
& +\left(\frac{T}{12}\right)^{\beta / 2} 2^{2+\beta} \mu M_{4}\left(2^{\beta-1} a_{4}^{\beta / 2}\left(C_{0} M_{1} h\left(\left|\bar{u}_{n}\right|\right)\right)^{\beta}\right. \\
& \left.+2^{3 \beta-2} \mu^{\beta / 2} M_{4}^{\beta / 2}\left|\bar{u}_{n}\right|^{\beta^{2} / 2}+2^{2(\beta-1)} C_{5}^{\beta}\right) \\
& +\left(\frac{T}{12}\right)^{1 / 2}\left(C_{0} M_{2} K_{2}+M_{3}\right)\left(\sqrt{a_{4}} C_{0} M_{1} h\left(\left|\bar{u}_{n}\right|\right)+2 \sqrt{\mu M_{4}}\left|\bar{u}_{n}\right|^{\beta / 2}+C_{5}\right) \\
& +M_{4}+\int_{0}^{T} F_{1}\left(t, \bar{u}_{n}\right) d t+\int_{0}^{T} F_{2}\left(\bar{u}_{n}\right) d t \\
& =h^{2}\left(\left|\bar{u}_{n}\right|\right)\left[h^{-2}\left(\left|\bar{u}_{n}\right|\right) \int_{0}^{T} F_{1}\left(t, \bar{u}_{n}\right) d t+\left(\frac{a_{4}}{2}+\frac{\sqrt{a_{4}} T}{2 \pi}\right)\left(C_{0} M_{1}\right)^{2}\right. \\
& +\left(\frac{T}{12}\right)^{(1+\alpha) / 2} C_{0}^{2+\alpha} M_{1}^{1+\alpha} M_{2} K_{1} 2^{\alpha} a_{4}^{(1+\alpha) / 2} h^{\alpha-1}\left(\left|\bar{u}_{n}\right|\right) \\
& +\left(\frac{T}{12}\right)^{\beta / 2} 2^{1+2 \beta} \mu\left(C_{0} M_{1}\right)^{\beta} M_{4} a_{4}^{\beta / 2} h^{\beta-2}\left(\left|\bar{u}_{n}\right|\right) \\
& \left.+\left(\frac{T}{12}\right)^{1 / 2}\left(C_{0} M_{2} K_{2}+M_{3}\right) \sqrt{a_{4}} C_{0} M_{1} h^{-1}\left(\left|\bar{u}_{n}\right|\right)\right] \\
& +\left|\bar{u}_{n}\right|^{\beta}\left[\left|\bar{u}_{n}\right|^{-\beta} \int_{0}^{T} F_{2}\left(\bar{u}_{n}\right) d t+\left(6+\frac{T}{\pi \sqrt{a_{4}}}\right) \mu M_{4}\right. \\
& +\left(\frac{T}{12}\right)^{(1+\alpha) / 2} 2^{3 \alpha+1} C_{0} M_{2} K_{1} \mu^{(1+\alpha) / 2} M_{4}^{(1+\alpha) / 2}\left|\bar{u}_{n}\right|^{\alpha \beta} \\
& +\left(\frac{T}{12}\right)^{\beta / 2} 2^{4 \beta} \mu^{(\beta+2) / 2} M_{4}^{(\beta+2) / 2}\left|\bar{u}_{n}\right|^{\frac{\beta^{2}}{2}-\beta} \\
& \left.+\left(\frac{T}{12}\right)^{1 / 2} 2\left(C_{0} M_{2} K_{2}+M_{3}\right) \sqrt{\mu M_{4}}\left|\bar{u}_{n}\right|^{-\beta / 2}\right] \\
& -\left(1+\frac{T}{2 \pi \sqrt{a_{4}}}\right) C_{4}+\left(\frac{T}{12}\right)^{(1+\alpha) / 2} 2^{2 \alpha} C_{0} M_{2} K_{1} C_{5}^{1+\alpha} \\
& +\left(\frac{T}{12}\right)^{1 / 2}\left(C_{0} M_{2} K_{2}+M_{3}\right) C_{5}+\left(\frac{T}{12}\right)^{\beta / 2} 2^{3 \beta} \mu M_{4} C_{5}^{\beta}+M_{4},
\end{aligned}
$$

for large $n$. The above inequality and (2.14) imply that $\{|\bar{u}|\}$ is bounded. Hence $\left\{u_{n}\right\}$ is bounded by (2.19). By using the standard method, the (PS) condition holds.

Since the rest of the proof is similar to that of Theorem 1.2, we omit the details here.

\section{Competing interests}

The authors declare that they have no competing interests.

\section{Authors' contributions}

The main idea of this paper was proposed by D-BW, D-BW prepared the manuscript initially, and KY performed a part of the steps of the proofs in this research. All authors read and approved the final manuscript. 
Acknowledgements

The author thanks the referees and the editors for their helpful comments and suggestions. The research was supported by NSFC (11561043)

Received: 1 July 2015 Accepted: 13 October 2015 Published online: 29 October 2015

\section{References}

1. Aizmahin, N, An, T: The existence of periodic solutions of non-autonomous second-order Hamiltonian systems. Nonlinear Anal. 74, 4862-4867 (2011)

2. Berger, MS, Schechter, M: On the solvability of semilinear gradient operator equations. Adv. Math. 25, 97-132 (1977)

3. Han, ZQ: $2 \pi$-periodic solutions to $n$-Duffing systems. In: Guo, DJ (ed.) Nonlinear Analysis and Its Applications, pp. 182-191. Beijng Scientific and Technical Publisher, Beijing (1994) (in Chinese)

4. Long, YM: Nonlinear oscillations for classical Hamiltonian systems with bi-even subquadratic potentials. Nonlinear Anal. 24, 1665-1671 (1995)

5. Mawhin, J: Semi-coercive monotone variational problems. Bull. Cl. Sci., Acad. R. Belg. 73, 118-130 (1987)

6. Mawhin, J, Willem, M: Critical Point Theory and Hamiltonian Systems. Springer, New York (1989)

7. Meng, Q, Tang, XH: Solutions of a second-order Hamiltonian system with periodic boundary conditions. Commun. Pure Appl. Anal. 9, 1053-1067 (2010)

8. Ma, J, Tang, CL: Periodic solutions for some nonautonomous second-order systems. J. Math. Anal. Appl. 275, 482-494 (2002)

9. Rabinowitz, PH: On subharmonic solutions of Hamiltonian systems. Commun. Pure Appl. Math. 33, 609-633 (1980)

10. Rabinowitz, PH: Minimax Methods in Critical Point with Applications to Differential Equations. CBMS, vol. 65. Am. Math. Soc., Providence (1986)

11. Tang, CL: Periodic solutions of nonautonomous second order systems with $\gamma$-quasisubadditive potential. J. Math. Anal. Appl. 189, 671-675 (1995)

12. Tang, CL: Periodic solutions of nonautonomous second order systems. J. Math. Anal. Appl. 202, $465-469$ (1996)

13. Tang, CL: Periodic solutions of nonautonomous second order systems with sublinear nonlinearity. Proc. Am. Math. Soc. 126, 3263-3270 (1998)

14. Tang, CL, Wu, XP: Periodic solutions for second order systems with not uniformly coercive potentia. J. Math. Anal. Appl. 259, 386-397 (2001)

15. Tang, $\mathrm{XH}$, Meng, Q: Solutions of a second-order Hamiltonian system with periodic boundary conditions. Nonlinear Anal., Real World Appl. 11, 3722-3733 (2010)

16. Willem, M: Oscillations forcées systèmes hamiltoniens. In: Public. Sémin. Analyse Non Linéaire. Université de Franche-Comté, Besancon (1981)

17. $\mathrm{Wu}, \mathrm{X}$ : Saddle point characterization and multiplicity of periodic solutions of nonautonomous second order systems. Nonlinear Anal. 58, 899-907 (2004)

18. $\mathrm{Wu}, \mathrm{XP}$, Tang, CL: Periodic solutions of a class of nonautonomous second order systems. J. Math. Anal. Appl. 236 227-235 (1999)

19. Zhao, F, Wu, X: Periodic solutions for a class of non-autonomous second order systems. J. Math. Anal. Appl. 296 422-434 (2004)

20. Zhao, F, Wu, X: Existence and multiplicity of periodic solution for non-autonomous second-order systems with linear nonlinearity. Nonlinear Anal. 60, 325-335 (2005)

21. Wang, ZY, Zhang, JH: Periodic solutions of a class of second order non-autonomous Hamiltonian systems. Nonlinea Anal. 72, 4480-4487 (2010)

22. $\mathrm{Wu}, \mathrm{Y}, \mathrm{An}, \mathrm{TQ}$ : Existence of periodic solutions for non-autonomous second-order Hamiltonian systems. Electron. J. Differ. Equ. 77, 1 (2013)

\section{Submit your manuscript to a SpringerOpen ${ }^{\circ}$ journal and benefit from:}

- Convenient online submission

Rigorous peer review

- Immediate publication on acceptance

- Open access: articles freely available online

- High visibility within the field

- Retaining the copyright to your article 